\title{
Myxofibroma of the maxilla. Reconstruction with iliac crest graft and dental implants after tumor resection
}

\author{
Pedro Infante-Cossío ${ }^{1}$, Rafael Martínez-de-Fuentes ${ }^{2}$, Alberto García-Perla-García ${ }^{3}$, Emilio Jiménez-Caste- \\ llanos $^{4}$, Lourdes Gómez-Izquierdo ${ }^{5}$
}

\author{
${ }^{1}$ Profesor Titular Vinculado, Servicio de Cirugía Oral y Maxilofacial, Hospital Universitario Virgen del Rocío, Universidad de \\ Sevilla \\ ${ }^{2}$ Profesor Asociado de Prótesis Estomatológica, Facultad de Odontología, Universidad de Sevilla \\ ${ }^{3}$ Profesor Asociado, Servicio de Cirugía Oral y Maxilofacial, Hospital Universitario Virgen del Rocío, Sevilla \\ ${ }^{4}$ Catedrático de Prótesis Estomatológica, Facultad de Odontología, Universidad de Sevilla \\ ${ }^{5}$ Facultativo Especialista del Servicio de Anatomía Patológica, Hospital Universitario Virgen del Rocío, Sevilla
}

\section{Correspondence:}

Servicio de Cirugia Oral y Maxilofacial Hospital Universitario Virgen del Rocío Av. Manuel Siurot. 41013-Sevilla, Spain pinfante@us.es

\author{
Infante-Cossío P, Martínez-de-Fuentes R, García-Perla-García A, Jimé- \\ nez-Castellanos E, Gómez-Izquierdo L. Myxofibroma of the maxilla. Re- \\ construction with iliac crest graft and dental implants after tumor resec- \\ tion. Med Oral Patol Oral Cir Bucal. 2011 Jul 1;16 (4):e532-6.

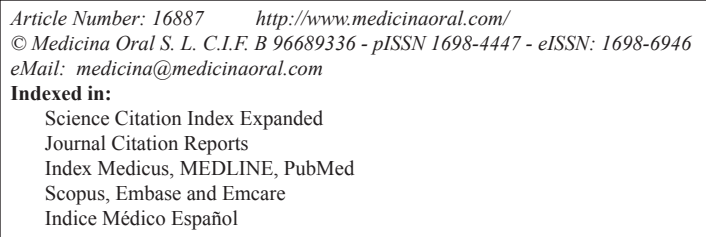

Received: 27/02/2010

Accepted: 06/04/2010

\begin{abstract}
Odontogenic fibromyxomas are benign odontogenic tumors of mesenchymal origin of rare presentation in the oral cavity, which exhibit locally aggressive behavior and are prone to local recurrence. The controversy has mainly been on therapeutic management with recommendations varying, depending on the clinical cases, from simple curettage of lesion to segmental bone resection. We present a case report describing the reconstruction of an osseous defect in the maxilla and the restoration with dental implants in a 32 year old female patient after radical surgical excision due to an odontogenic fibromyxoma with locally aggressive behavior. The primary reconstruction of maxillary discontinuity defect was carried out by an immediate non-vascularized cortico-cancellous iliac crest graft. Using a computer-guided system for the implant treatment-planning, three dental implants were secondary placed in the bone graft by means of flapless implant surgery. The patient was subsequently restored with an implant-supported fixed prosthesis that has remained in continuous function for a period of three years. The surgical, reconstructive and restorative treatment sequence and techniques are discussed.
\end{abstract}

Key words: Odontogenic fibromyxoma, maxilla, reconstruction, dental implants, computer-assisted surgery. 


\section{Introduction}

Odontogenic fibromyxomas are rare benign odontogenic tumors of mesenchymal origin. They occur more frequently between 10 and 40 years of age with an incidence of approximately 0.07 new cases per million people per year (1). They are more common in females and located mainly in the mandible. Histologically they are composed of large amounts of intercellular substance rich in acid mucopolysaccharides and made up of loose myxomatous connective tissue, fibroblasts and myofibroblasts. Patches of trabeculae of woven bone and capillaries are dispersed by the lesion (2).

There is a wide variety in clinical and radiologic appearance of odontogenic fibromyxomas, being the most common form of presentation as an asymptomatic expansion in the jaw and a multilocular radiolucent image. Although they are a slow-growing lesion, they show a persistent and destructive pattern. As they grow they usually displace and resorb teeth causing pain and facial deformity. In the maxilla, they have the potential of destroying bone extensively and invading the maxillary sinuses. Its histological and radiological features make it difficult to differentiate from other odontogenic tumors and occasionally may be misinterpreted as a malignant lesion.

Odontogenic fibromyxomas exhibit locally aggressive behavior and are prone to local recurrence. The controversy has mainly been on therapy with recommendations varying from simple curettage of the lesion to radical surgery. Although curettage of the lesion is used for treatment, it is associated with a high recurrence rate up to $25 \%$ (3). It typically occurs during the first 2 years after removal (4). The treatment of choice in large, destructive and expansile lesions is surgical removal with safety margins of at least $1.5 \mathrm{~cm}$ to prevent recurrence of the neoplasm. In most cases, tumor ablation usually involves the sacrifice of adjacent teeth.

The reconstruction of patients with continuity defects of the maxilla after tumor resection including the loss of several teeth is very challenging. The basic method for providing bone with osteogenic capacity in the maxillary area is carried out by non-vascularized bone grafts. Autogenous bone grafts taken from the iliac crest have demonstrated to be a very reliable means for the reconstruction of maxillofacial defects after ablation of tumors (5). Subsequent to the osseous anatomic reconstruction, masticatory function of missing teeth can be achieved by placing dental implants and using implant-supported prosthesis. When raising a flap during the implant surgery in patients who have previously undergone a bone reconstruction with iliac crest graft, one of the main risks is often damage to the graft vascularization from periostium, thus producing an unpredictable bone resorption.

The purpose of the present article is to report an unusual case of an aggressive fibromyxoma in the maxilla, with emphasis on the surgical treatment, reconstruction of maxillary discontinuity defect with an iliac crest graft, and restoration with an implant-supported prosthesis using a computer-guided system for the treatment-planning and precise placement of dental implants by means of flapless surgery.

\section{Case Report}

A 32-year-old woman presented to consultation in February 2006 with a history of swelling in the anterior maxillary region. Three months before the patient had felt mobility in the maxillary right canine and first premolar. From then on, a swelling involving the buccal vestibule in the anterior maxilla and palate was growing gradually, although it caused her no pain. Intraoral examination revealed an expansive bony mass involving the buccal maxillary right canine region, extending to the area of the maxillary right lateral incisive and first premolar, also affecting palate but less evident. On palpation the swelling had hard consistency, and buccal and palatal cortices were expanded. Panoramic radiograph showed a $2 \times 1 \mathrm{~cm}$ well-demarcated radiolucent lesion of multiloculated appearance involving the anterior right maxilla, and root resorption in the right maxillary canine, first premolar and lateral incisive (Fig. 1a). In computed tomography (CT), an expansible $2 \times 1 \times 1 \mathrm{~cm}$ lesion was visualized perforating and destroying the buccal and palatal cortical bone, homogeneous hypodensity with peripheral septi and an aggressive radiological pattern (Fig. 1b). The lesion was in close relation with

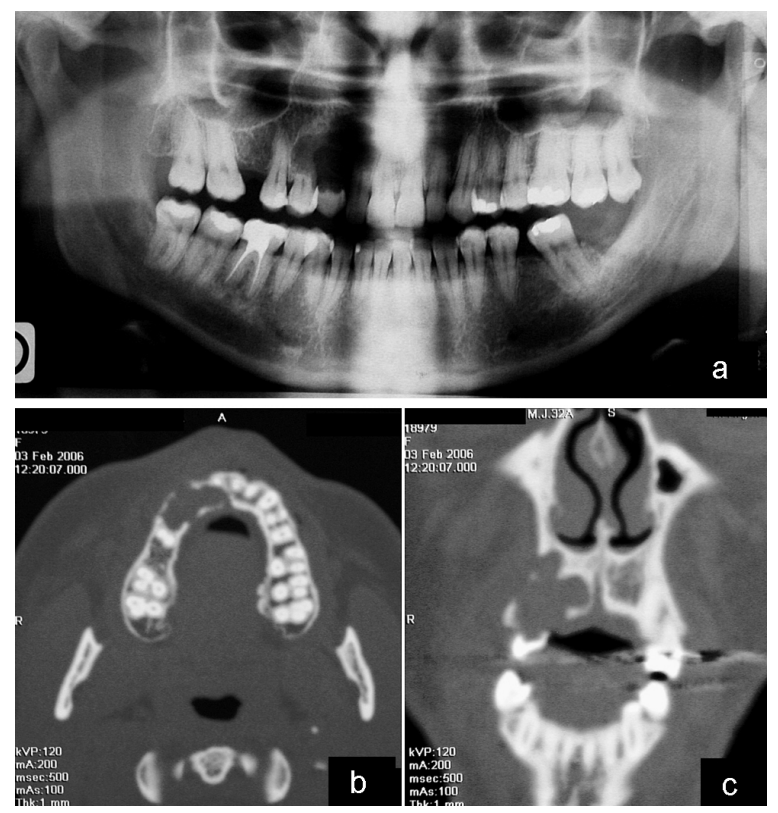

Fig. 1. 1a. Panoramic radiograph showing a radiolucent image of multiloculated appearance involving the maxilla, and root resorption in the right maxillary canine, first premolar and lateral incisive. 1b. Axial CT showing an expansive and osteolytic lesion destroying the buccal and palatal cortical bone. 1c. Coronal CT demonstrating an aggressive radiological pattern in close relation with the maxillary sinus and nasal cavity. 
the maxillary sinus and nasal cavity (Fig. 1c). Intraoral incisional biopsy was performed under local anesthesia, which revealed a collection of fibroblastic tissue and islands of odontogenic epithelium with calcium. The diagnosis was compatible with odontogenic fibromyxoma.

Under general anesthesia the lesion was removed en bloc via an intraoral approach with bone security margins, along with the involved teeth from maxillary right central incisive through first premolar. The surgical specimen consisted of a partial segmental maxillectomy just above the floor of the nasal cavity and maxillary sinus (Fig. 2a). After removal of the lesion, the surgical defect received an immediate reconstruction using a cortico-cancellous anterior iliac crest graft (Fig. 2b), and two miniplates were fixed to stabilize it. Soft tissue closure was achieved by local flaps. The patient was discharged from the hospital on the third postoperative day. There were no postoperative complications. Histological examination of the excised lesion showed abundantly myxoid connective stroma, areas of moderately dense collagen fibers, and strands of odontogenic epithelium within the connective tissue stroma. The presence of myxomatous tissue, along with areas of moderately dense collagen fibers, led to the final diagnosis of myxofibroma odontogenic (Fig. 2c).

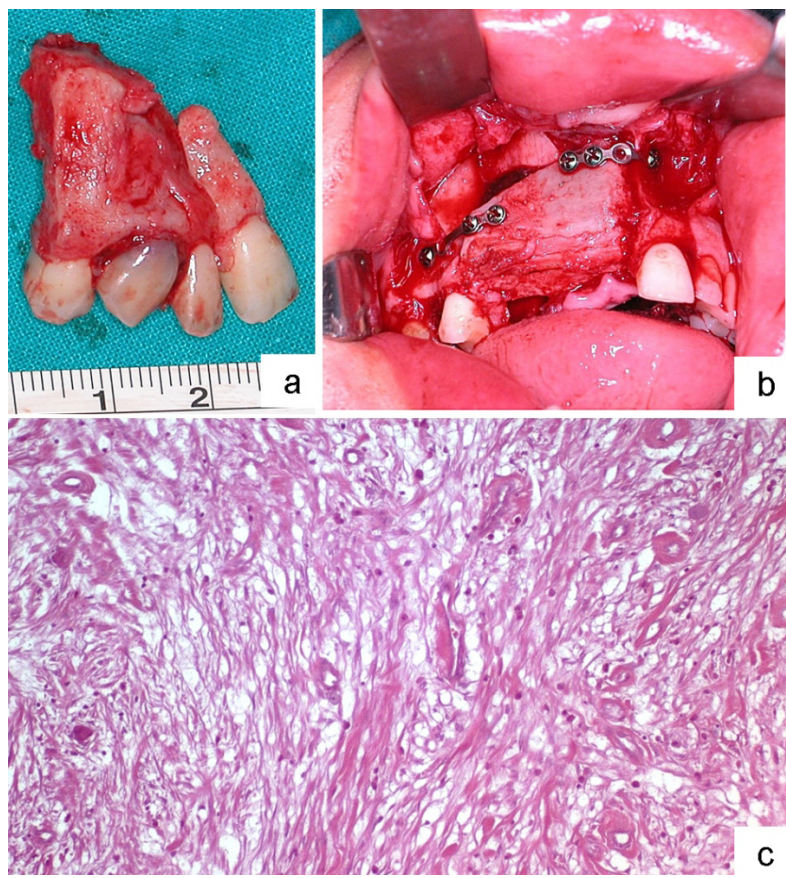

Fig. 2. 2a. Surgical specimen of the maxilla including the involved teeth. 2b. Maxillary defect after tumor resection and immediate reconstruction using a cortico-cancellous iliac crest graft. 2c. Abundant myxoid stroma, areas of moderately dense collagen fibers, and strands of odontogenic epithelium within a connective tissue stroma (hematoxylin and eosin 100x).

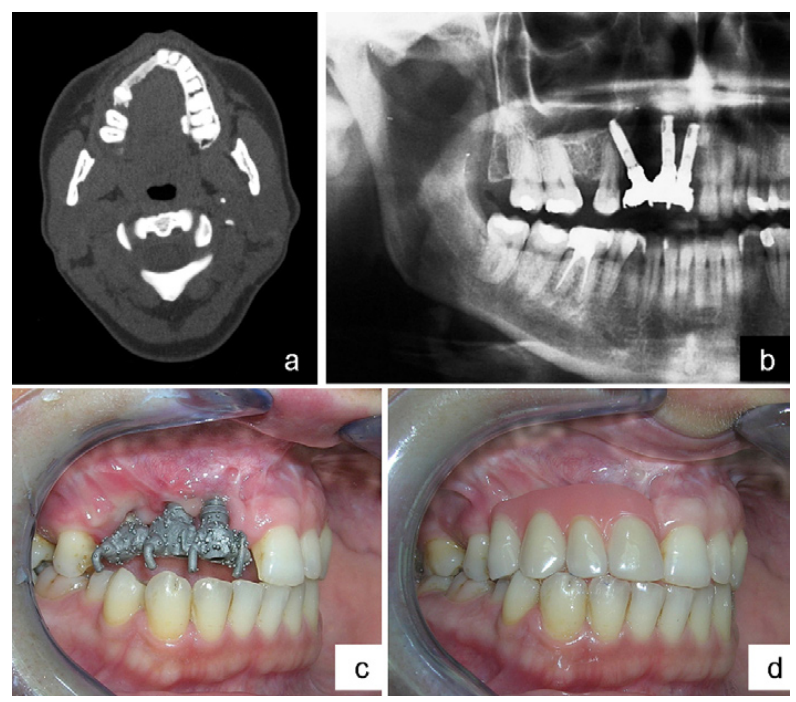

Fig. 3. 3a. Axial CT showing a well-integration of the bone graft. 3b. Panoramic radiograph of the 3 implants placed in the graft three years later without recurrence of lesion. $3 \mathrm{c}$. Intraoral view of the reconstruction with the implants and the structure for the prosthesis. $3 \mathrm{~d}$. View of the partial implant-supported prosthesis.

Six months post-surgery, a panoramic radiograph and CT was performed confirming complete integration of the graft, with no evidence of tumor recurrence (Fig. 3a). For miniplates retrieval, a re-entry was performed through a minimal incision in the vestibule. Two weeks later, an osseus-dento-suported surgery template was planned and constructed using the NobelGuide ${ }^{\circledR}$ programme (Nobel Biocare ${ }^{\circledR}$ AB, Gothenburg, Sweden). One anchor pin was used to provide additional stabilization of the surgical template. Via the guided surgical template, two Bränemark Systems MKIII TiUnite implants $3.75 \mathrm{~mm}$ in diameter and $13 \mathrm{~mm}$ long and one Nobelspeedy Groovy NP $3.3 \times 13 \mathrm{~mm}$ (Nobel Biocare ${ }^{\circledR}$ AB) were inserted in the iliac crest graft through a minimally invasive flapless implant surgery. After placement of implants, the guided surgical template was removed, and healing abutments screwed. Primary stability of implants was achieved. Three months later, a partial implant-supported prosthesis made of metalresin was constructed. After 3 years, no recurrence of the tumor has occurred. The patient is being reviewed in periodic checks. Follow up must be performed once a year up to 10 years. The patient has a high level of comfort and satisfaction (Figs. 3b, c and d).

\section{Discussion}

The therapeutic approach to the fibromyxoma is still today a controversy. The treatment of choice consists of surgical resection. However, no unanimous consensus exists concerning the extension of surgery. A more conservative approach is normally considered for small 
lesions which can be treated with curettage and simple enucleation (6). An alternative approach may include the addition of a peripheral ostectomy to remove a circumferential margin of bone around the tumour. Larger tumours often require more extensive resection as recurrence rates are as high as $25 \%$ (3). The absence of capsule and infiltrative growth of the myxomatous tissue to the adjacent bone determine the high rate of recurrence when conservative enucleation and curettage are performed (1). Recurrence is the result of local invasion into cancellous bone beyond radiographically visible margins in the absence of encapsulation (4). Recurrence is minimized with a more aggressive treatment by performing a partial or complete segmental bone resection, which is particularly indicated in the maxilla. Therefore, a radical resection including an osseous margin of at least $1.5-2 \mathrm{~cm}$ seems to be the best option to prevent recurrences of large fibromyxomas (4).

In our case, an aggressive approach was chosen due to the fact that a local destructive behavior was encountered (perforation of cortical bone, important tumour expansion, and infiltration in a short period of time). We performed a partial segmental maxillectomy, which consisted of an en bloc resection of the tumor with surgical margins beyond the radiographic margins of bone, up to the edge of the maxillary sinus and nasal cavity that were preserved (7).The possibility of carrying out a primary maxillary reconstruction with optimal results both aesthetic and functional encouraged us to choose this option, moreover if in a second stage we could place dental implants and restore with an implant-supported prosthesis. Although the tendency towards recurrence after this radical resection is very low, postoperative follow-up of our patient for 10 years is advisable.

Bone reconstruction can be immediate or delayed following the surgical procedure, and can be performed with autogenous bone grafts from iliac crest (8) or microvascularized free flaps (9). In our experience, small maxillary bone defects (smaller than $5 \mathrm{~cm}$ ) can be reconstructed using cortico-cancellous iliac crest bone graft (7). Iliac crest can be recommended in almost all reconstructive situations because it reinforces the resistance of the maxilla, re-establishes its continuity improving facial contour, and provides adequate height and width of bone. Once the graft has consolidated, these reconstructions have proved to be very acceptable aesthetically and functionally to support dental prostheses or for the insertion of dental implants $(5,9)$.

However, iliac crest bone grafts present the major problem of its unpredictable resorption. The capacity for resorption is low if the graft is covered adequately by the periosteum of the flap, which promotes the re-vascularization and incorporation of the transplanted bone from an early stage. An additional problem occurs in the maxilla if the graft keeps in contact and communication with natural cavities, as the resorption of the graft may be higher due to compromised re-vascularization and exposition to possible infection. Many publications in the last few years have reported very good results with the use of implants and implant-supported prostheses in reconstructed patients after tumor resection, and demonstrated that they seem to reduce bone resorption significantly, probably due to the fact that prosthetic load stimulates the graft and inhibits bone resorption $(5,10)$. The period of time of delay between bone graft reconstruction and implant placement can fluctuate from 4 to 6 months. This waiting time would allow us to verify the consolidation and re-vascularization of the grafted bone, decreasing the risk of excessive graft resorption. When placing implants, one of the significant difficulties posed is to ensure the proper positioning within the reconstructed alveolar ridge. In our patient, it was the authors' opinion that a minimally invasive flapless surgical technique would have several benefits compared to the conventional surgical procedure of raising a flap before implant insertion. Flapless surgery would not only generate less postoperative bleeding and minor swelling (11), but also less damage to the vascularization of the graft as the periostium would remain intact.

The importance of dental implant treatment planning using 3-D CT-scan is increasing. Computer-guided surgery allows greater accuracy in determining the number of implants, their location and positioning, offers more advantages to use the amount of available bone and improve stability in soft bone, and also facilitates minimally invasive surgery (12). It is a very suitable procedure to insert implants with precision in selected locations and areas with anatomical limitations, such as the inferior alveolar nerve, nasal cavity or maxillary sinus (13). Its usefulness has been also demonstrated in the treatment of atrophic maxilla. However, to the best of our knowledge, the use of a computer-guided system for the treatment-planning and subsequent accurate placement of dental implants by means of flapless surgery has been no reported in a patient who previously had undergone a reconstruction of the maxilla with an iliac crest graft.

The planning procedure through a computerized method based on 3-D CT-scan images made it possible to preview in advance the more favorable implant location, and the fabrication of a customized guided surgical template (14). The combined use of a software planning program and a customized template allowed the placement of implants by means of a flapless surgery (NobelGuide ${ }^{\circledR}$ ) without raising a mucoperiostal flap (15), which enabled a surgical procedure where only it took a small opening of the mucosa for the optimal positioning of implants with precision. This treatment sequence had several advantages. It made it possible the safe insertion of the implants according to bone volume and without 
intruding any anatomical cavities; the procedure provided minimal post-operative discomfort to the patient by reducing swelling and pain (5); and by omission of raising the mucoperiosteal flap, the postoperative bone resorption potentially associated with the procedures of full-thickness mucoperiosteal flap was prevented.

\section{References}

References with links to Crossref - DOI

1. Simon EN, Merkx MA, Vuhahula E, Ngassapa D, Stoelinga PJ. Odontogenic myxoma: a clinicopathological study of 33 cases. Int J Oral Maxillofac Surg. 2004;33:333-7.

2. Boleaga-Durán B, Palacios E. Odontogenic myxoma of the maxilla. Ear Nose Throat J. 2007:86:444,446.

3. Lo Muzio L, Nocini P, Favia G, Procaccini M, Mignogna MD. Odontogenic myxoma of the jaws: a clinical, radiologic, immunohistochemical, and ultrastructural study. Oral Surg Oral Med Oral Pathol Oral Radiol Endod. 1996;82:426-33.

4. King TJ 3rd, Lewis J, Orvidas L, Kademani D. Pediatric maxillary odontogenic myxoma: a report of 2 cases and review of management. J Oral Maxillofac Surg. 2008;66:1057-62.

5. Chiapasco M, Colletti G, Romeo E, Zaniboni M, Brusati R. Longterm results of mandibular reconstruction with autogenous bone grafts and oral implants after tumor resection. Clin Oral Implants Res. 2008;19:1074-80.

6. Hernández Vallejo G, Cohn C, García Peñín A, Martínez Lara S, Llanes Menéndez F, Montalvo Moreno JJ. Myxoma of the jaws. Report of three cases. Med Oral. 2001;6:106-13.

7. Leiser Y, Abu-El-Naaj I, Peled M. Odontogenic myxoma--a case series and review of the surgical management. J Craniomaxillofac Surg. 2009;37:206-9.

8. Schneck DL, Gross PD, Tabor MW. Odontogenic myxoma: report of two cases with reconstruction considerations. J Oral Maxillofac Surg. 1993;51:935-40.

9. Chiapasco M, Abati S, Ramundo G, Rossi A, Romeo E, Vogel G. Behavior of implants in bone grafts or free flaps after tumor resection. Clin Oral Implants Res. 2000;11:66-75.

10. Pogrel MA, Podlesh S, Anthony JP, Alexander J. A comparison of vascularized and nonvascularized bone grafts for reconstruction of mandibular continuity defects. J Oral Maxillofac Surg. 1997;55:1200-6.

11. Rocci A, Martignoni M, Gottlow J. Immediate loading in the maxilla using flapless surgery, implants placed in predetermined positions, and prefabricated provisional restorations: a retrospective 3-year clinical study. Clin Implant Dent Relat Res. 2003;5 Suppl 1:29-36.

12. Rubio Serrano M, Albalat Estela S, Peñarrocha Diago M, Peñarrocha Diago M. Software applied to oral implantology: update. Med Oral Patol Oral Cir Bucal. 2008;13:E661-5.

13. Engelke W, Capobianco M. Flapless sinus floor augmentation using endoscopy combined with CT scan-designed surgical templates: method and report of 6 consecutive cases. Int J Oral Maxillofac Implants. 2005;20:891-7.

14. Casap N, Wexler A, Tarazi E. Application of a surgical navigation system for implant surgery in a deficient alveolar ridge postexcision of an odontogenic myxoma. J Oral Maxillofac Surg. 2005;63:982-8. 15. Parel SM, Triplett RG. Interactive imaging for implant planning, placement, and prosthesis construction. J Oral Maxillofac Surg. 2004:62:41-7. 